\title{
A new measurement of the $\Xi_{c}^{0}$ lifetime
}

\author{
The FOCUS Collaboration *
}

J. M. Link ${ }^{a}$ M. Reyes ${ }^{a}$ P. M. Yager ${ }^{a}$ J. C. Anjos ${ }^{b} I_{\text {. Bediaga }}{ }^{b}$ C. Göbel ${ }^{b}$ J. Magnin ${ }^{b}$ A. Massafferri ${ }^{b}$ J. M. de Miranda ${ }^{b}$ I. M. Pepe ${ }^{b}$ A. C. dos Reis ${ }^{b}$ S. Carrillo ${ }^{c}$ E. Casimiro ${ }^{c}$ E. Cuautle ${ }^{c}$ A. Sánchez-Hernández ${ }^{c}$ C. Uribe ${ }^{c}$ F. Vázquez ${ }^{c}$ L. Agostino ${ }^{d}$ L. Cinquini ${ }^{d}$ J. P. Cumalat ${ }^{d}$ B. O'Reilly ${ }^{d}$ J. E. Ramirez ${ }^{\text {d }}$ I. Segoni $^{\text {d }}$ J. N. Butler ${ }^{e}$ H. W. K. Cheung ${ }^{\mathrm{e}}$ G. Chiodini ${ }^{\text {e }}$ I. Gaines ${ }^{\text {e }}$ P. H. Garbincius ${ }^{\text {e }}$ L. A. Garren ${ }^{\text {e }}$ E. Gottschalk ${ }^{\mathrm{e}}$ P. H. Kasper ${ }^{\mathrm{e}}$ A. E. Kreymer ${ }^{\mathrm{e}}$ R. Kutschke ${ }^{\mathrm{e}}$ L. Benussi ${ }^{f}$ S. Bianco ${ }^{f}$ F. L. Fabbri ${ }^{f}$ A. Zallo ${ }^{f}$ C. Cawlfield ${ }^{g}$ D. Y. Kim ${ }^{g}$ K. S. Park ${ }^{g}$ A. Rahimi ${ }^{g}$ J. Wiss ${ }^{g}$ R. Gardner ${ }^{\mathrm{h}}$ A. Kryemadhi ${ }^{\text {h }}$ K. H. Chang ${ }^{i}$ Y. S. Chung ${ }^{i}$ J. S. Kang ${ }^{i}$ B. R. Ko ${ }^{i}$ J. W. Kwak ${ }^{i}$ K. B. Lee ${ }^{i}$ K. Cho ${ }^{j}$ H. Park ${ }^{j}$ G. Alimonti ${ }^{k}$ S. Barberis ${ }^{k}$ A. Cerutti ${ }^{k}$ M. Boschini ${ }^{k}$ P. D'Angelo ${ }^{k}$ M. DiCorato ${ }^{k}$ P. Dini ${ }^{k}$ L. Edera ${ }^{k}$ S. Erba ${ }^{k}$ M. Giammarchi ${ }^{k}$ P. Inzani ${ }^{k}$ F. Leveraro ${ }^{k}$ S. Malvezzi ${ }^{k}$ D. Menasce ${ }^{k}$ M. Mezzadri ${ }^{k}$ L. Moroni ${ }^{k}$ D. Pedrini ${ }^{k}$ C. Pontoglio ${ }^{k}$ F. Prelz ${ }^{k}$ M. Rovere ${ }^{k}$ S. Sala ${ }^{k}$

T. F. Davenport III $^{\ell}$ V. Arena ${ }^{\mathrm{m}}$ G. Boca ${ }^{\mathrm{m}}$ G. Bonomi ${ }^{\mathrm{m}}$ G. Gianini ${ }^{\mathrm{m}}$ G. Liguori ${ }^{\mathrm{m}}$ M. M. Merlo ${ }^{\mathrm{m}}$ D. Pantea ${ }^{\mathrm{m}}$ S. P. Ratti ${ }^{m}$ C. Riccardi ${ }^{m}$ P. Vitulo ${ }^{m}$ H. Hernandez ${ }^{n}$ A. M. Lopez ${ }^{n}$ H. Mendez ${ }^{n}$ L. Mendez ${ }^{n}$ E. Montiel ${ }^{n}$ D. Olaya ${ }^{n}$ A. Paris ${ }^{n}$ J. Quinones ${ }^{n}$ C. Rivera ${ }^{n}$ W. Xiong ${ }^{n}$ Y. Zhang ${ }^{n}$ J. R. Wilson ${ }^{\mathrm{o}}$ T. Handler ${ }^{\mathrm{p}}$ R. Mitchell ${ }^{\mathrm{p}}$ D. Engh ${ }^{\mathrm{q}}$ M. Hosack ${ }^{\mathrm{q}}$ W. E. Johns ${ }^{q}$ M. Nehring ${ }^{q}$ P. D. Sheldon ${ }^{q}$ K. Stenson ${ }^{q}$ E. W. Vaandering ${ }^{q}$ M. Webster ${ }^{q}$ M. Sheaff ${ }^{r}$

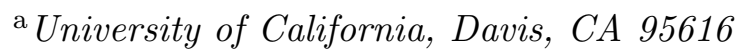

${ }^{\mathrm{b}}$ Centro Brasileiro de Pesquisas Físicas, Rio de Janeiro, RJ, Brasil

${ }^{\mathrm{c}}$ CINVESTAV, 07000 México City, DF, Mexico

d University of Colorado, Boulder, CO 80309

\footnotetext{
ऋ see http://www-focus.fnal.gov/authors.html for additional author information.
} 
${ }^{\text {e }}$ Fermi National Accelerator Laboratory, Batavia, IL 60510

${ }^{\mathrm{f}}$ Laboratori Nazionali di Frascati dell'INFN, Frascati, Italy I-00044

${ }^{\mathrm{g}}$ University of Illinois, Urbana-Champaign, IL 61801

${ }^{\mathrm{h}}$ Indiana University, Bloomington, IN 47405

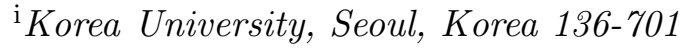

${ }^{\mathrm{j}}$ Kyungpook National University, Taegu, Korea 702-701

${ }^{\mathrm{k}}$ INFN and University of Milano, Milano, Italy

${ }^{\ell}$ University of North Carolina, Asheville, NC 28804

${ }^{\mathrm{m}}$ Dipartimento di Fisica Nucleare e Teorica and INFN, Pavia, Italy

${ }^{\mathrm{n}}$ University of Puerto Rico, Mayaguez, PR 00681

${ }^{\circ}$ University of South Carolina, Columbia, SC 29208

${ }^{\mathrm{p}}$ University of Tennessee, Knoxville, TN 37996

${ }^{\mathrm{q}}$ Vanderbilt University, Nashville, TN 37235

${ }^{\mathrm{r}}$ University of Wisconsin, Madison, WI 53706

\begin{abstract}
Using data collected by the Fermilab experiment FOCUS, we measure the lifetime of the charmed baryon $\Xi_{c}^{o}$ using the decay channels $\Xi_{c}^{o} \rightarrow \Xi^{-} \pi^{+}$ and $\Xi_{c}^{o} \rightarrow \Omega^{-} K^{+}$. From a combined sample of $110 \pm 17$ events we find $\tau\left(\Xi_{c}^{o}\right)=118_{-12}^{+14} \pm 5$ fs, where the first and second errors are statistical and systematic, respectively.
\end{abstract}

\title{
1 Introduction
}

While the lifetime hierarchy of the weakly decaying charm mesons is well established experimentally [1] and in fairly good agreement with theoretical calculations [2], the pattern of lifetimes in the charm baryon sector still needs to be established both experimentally and theoretically [3]. While the $\Lambda_{c}^{+}$ lifetime is known to an accuracy of about $2 \%$ [4-6], and recently the $\Xi_{c}^{+}$ lifetime has been determined to an accuracy of $5 \%$ [7], the $\Xi_{c}^{0}$ and $\Omega_{c}^{0}$ lifetimes remain known to only $20 \%$ and $30 \%$, respectively.

The lifetime of the $\Xi_{c}^{0}$ has been measured by two experiments. CERN experiment NA32 found a lifetime of $82_{-30}^{+59}$ fs using four events reconstructed in the decay channel $\Xi_{c}^{0} \rightarrow p K^{-} \bar{K}^{* 0}$ [8]. Fermilab experiment E687 [9] measured a $\Xi_{c}^{0}$ lifetime [10] of $101_{-17}^{+25}$ (stat) \pm 5 (sys) fs using 42 events from the decay $\Xi_{c}^{0} \rightarrow \Xi^{-} \pi^{+}$. 
FOCUS (Fermilab E831) is a general purpose experiment investigating charm physics. The charmed particles are produced by the interaction of a photon beam on a segmented $\mathrm{BeO}$ target. The average energy of the beam for the data collected for this measurement is about $180 \mathrm{GeV}$. The track reconstruction is accomplished by two silicon vertex detectors [11,12] (TS and SSD) in the target region and by five multi-wire proportional chambers (MWPC) downstream of the target region. The charged momenta are determined by a measurement of the bending angles in two magnets of opposite polarity. Due to the excellent separation between production and decay vertices provided by the silicon detectors, we achieve an average proper time resolution of about 50 fs for the decay channels used in this analysis. Charged particle identification of hadrons is performed with three multi-cell threshold Cerenkov counters [13].

\section{Reconstruction of hyperons $\Xi^{-}$and $\Omega^{-}$}

We reconstruct the decay modes $\Xi_{c}^{0} \rightarrow \Xi^{-} \pi^{+}$and $\Xi_{c}^{o} \rightarrow \Omega^{-} K^{+}$. A detailed description of the reconstruction method and the mass spectra of the hyperons $\Xi^{-}$and $\Omega^{-}$is in Reference [14]. We reconstruct the $\Xi^{-}$decay channel $\Lambda^{0} \pi^{-}$and the $\Omega^{-}$decay channel $\Lambda^{0} K^{-}$. While these decay topologies are very similar, we can easily distinguish between them using Cerenkov identification of the final state particles and by requirements of the reconstructed invariant masses. We reconstruct the entire decay chain of these two modes. The $\Lambda^{0}$ is identified by its decay into a proton and an oppositely charged pion. The tracks of these charged daughters are used to form the decay vertex of the $\Lambda^{0}$ and to determine its flight direction and momentum. This direction is used together with the momentum of either the $\pi^{-}$or the $K^{-}$, to determine the decay vertex and momentum of the hyperon. The hyperon vertex must lie upstream of the $\Lambda^{0}$ vertex. Finally, the position and momentum vector of the hyperon is matched to a SSD track.

For both modes we compute the invariant mass of the two body combination and select the events which are in the correct hyperon mass region. For the $\Xi^{-}$we require $1.312 \mathrm{GeV} / c^{2}<M\left(\Lambda^{0} \pi^{-}\right)<1.330 \mathrm{GeV} / c^{2}$ and for the $\Omega^{-}$we require $1.665 \mathrm{GeV} / c^{2}<M\left(\Lambda^{0} K^{-}\right)<1.680 \mathrm{GeV} / c^{2}$.

\section{Reconstruction of $\Xi_{c}^{0}$ candidates}

The $\Xi_{c}^{0}$ candidates are reconstructed using a candidate driven vertexing algorithm [9]. Briefly, combinations of tracks are used to form a secondary vertex which in turn must point within errors to a primary production vertex. Pairs 
of $\Xi^{-}\left(\Omega^{-}\right)$candidates and oppositely charged pions (kaons) are intersected to form a secondary vertex and a $\Xi_{c}^{0}$ mass. Several criteria are used to reject the background contributions. Any track consistent with an $e^{+} e^{-}$pair hypothesis is rejected. A confidence level (CLS) for the secondary vertex hypothesis from the intersection of the two tracks is calculated, and a minimum cut is applied, the magnitude of which depends on the decay mode. The $\Xi_{c}^{0}$ decay products are used to construct the $\Xi_{c}^{0}$ momentum vector, which is combined with at least two additional silicon tracks to form the primary vertex, which must lie within the target region. A confidence level (CLP) for the primary vertex is calculated, and the combination is rejected if CLP $<1 \%$.

We require a minimum value for the significance of the separation between the secondary and primary vertex, given by $\ell / \sigma_{\ell}$, where $\ell$ is the distance between the two vertices and $\sigma_{\ell}$ is the uncertainty on $\ell$. The Cerenkov particle identification uses requirements on the variables $\mathrm{W}_{e}, \mathrm{~W}_{\pi}, \mathrm{W}_{K}$, and $\mathrm{W}_{p}$, which represent the negative log likelihood for the hypothesis that a particular track is an electron, a pion, a kaon, or a proton, respectively. The difference between two of these variables represents the ratio between the two probabilities. The pion consistency of a track is defined by a requirement on the variable picon $=W_{\min }-W_{\pi}$, where $W_{\min }$ is the minimum likelihood of the four corresponding hypotheses. The specific requirements for the two decay modes are now discussed.

For the $\Xi^{-} \pi^{+}$mode, we apply the detachment cut $\ell / \sigma_{\ell}>3$ and we require CLS $>3 \%$. During the data collection the tracking system was improved with the introduction of a silicon vertex detector in the target region; we require $\sigma_{t}<150$ fs and $\sigma_{t}<80$ fs where $\sigma_{t}$ is the error on the decay time of the $\Xi_{c}^{0}$, respectively, for data collected before and after the introduction of this detector. The pion (decay product of the $\Xi_{c}^{0}$ ) is required to have picon $>-10$ and a momentum greater than $7 \mathrm{GeV} / \mathrm{c}$.

For the $\Omega^{-} K^{+}$mode, the detachment cut is $\ell / \sigma_{\ell}>0.5$. For the secondary vertex we require CLS $>1 \%$. To identify kaons, we require $W_{p}-W_{K}>-0.1$ and $W_{\pi}-W_{K}>1$, comparing the kaon hypothesis to the proton and the pion hypothesis. Since $\Xi^{-}$particles are produced more copiously than $\Omega^{-}$ particles, we compute the invariant mass for the $\Omega^{-}$when it is calculated as a $\Lambda^{0} \pi^{-}$combination. To eliminate $\Xi^{-}$contamination under the $\Omega^{-}$mass, we require $M\left(\Lambda^{0} \pi^{-}\right)>1.375 \mathrm{GeV} / c^{2}$.

Figure 1 shows the invariant mass plots for the two modes. The fit is to a Gaussian for the signal and a straight line for the background. We do not use the fit information to perform the lifetime measurement. 


\section{The lifetime measurement}

We use a binned maximum likelihood fit method to measure the lifetime. The fitted histogram is the reduced proper time distribution: $t^{\prime}=\left(\ell-N \sigma_{\ell}\right) /(\beta \gamma c)$, where $N$ is the vertex detachment cut, and $\beta \gamma=p\left(\Xi_{c}^{0}\right) / M\left(\Xi_{c}^{0}\right)$. The fit is performed on the data in the signal region of the invariant mass distribution: the mass window within $\pm 2 \sigma$ of the $\Xi_{c}^{0}$ nominal mass, where $\sigma$ is the width of the Gaussian fit of the invariant mass distribution. The $t^{\prime}$ distribution of the signal region differs from the expected behavior $e^{-t^{\prime} / \tau}$ due to the presence of background events. We assume that the background events in the signal region have the same time distribution as the events of the sidebands (located between 5 and $9 \sigma$ away from the $\Xi_{c}^{0}$ mass). If $S$ is the total number of signal events in the signal region, and $B$ is the total number of background events in the signal region, the expected number of events $n_{i}$ in the $i^{\text {th }}$ bin of the $t^{\prime}$ distribution is:

$$
n_{i}=S \frac{f\left(t_{i}^{\prime}\right) e^{-t_{i}^{\prime} / \tau}}{\sum_{i} f\left(t_{i}^{\prime}\right) e^{-t_{i}^{\prime} / \tau}}+B \frac{b_{i}}{\sum_{i} b_{i}}
$$

where $b_{i}$ is the number of events in the $i^{\text {th }}$ bin of the sideband $t^{\prime}$ distribution. The Monte Carlo correction function $f\left(t^{\prime}\right)$ takes into account detector acceptance, the efficiency of the selection cuts, and absorption of the daughter particles as a function of the reduced proper time. Figure 2 shows the correction functions for the two modes; due to the lower detachment cut we observe a bigger correction for the $\Omega^{-} K^{+}$mode. Figure 3 shows the reduced proper time distribution for each mode after the Monte Carlo correction. For each mode a likelihood is constructed as the product between the Poisson probability of observing $s_{i}$ events in the signal region, when $n_{i}$ are expected, and the Poisson probability of observing $\sum_{i} b_{i}$ background events when $B$ are expected. A factor of 2 is included because the sidebands are twice as wide as the signal region. The likelihood for each category is thus:

$$
L=\left(\Pi_{i} \frac{n_{i}^{s_{i}} e^{-n_{i}}}{s_{i} !}\right) \times\left(\frac{(2 B)^{\sum_{i} b_{i}} e^{-2 B}}{\left(\sum_{i} b_{i}\right) !}\right)
$$

To measure $\tau$, we maximize the combined likelihood, which is the product of the likelihoods for the two decay modes:

$$
L_{\Xi_{c}^{0}}=L_{\Xi \pi} \times L_{\Omega K}
$$

There are three fit parameters: the lifetime $\tau$, and for each mode the background events $B$. Our fit result is $\tau=118_{-12}^{+14}$ fs. When fitted separately, we measure for the two decay modes $\tau\left(\Xi_{c}^{0} \rightarrow \Xi^{-} \pi^{+}\right)=117_{-13}^{+15}$ fs and $\tau\left(\Xi_{c}^{0} \rightarrow\right.$ $\left.\Omega^{-} K^{+}\right)=122_{-25}^{+37}$ fs. 
Table 1

\begin{tabular}{cc}
\hline \hline Contribution & Systematic (fs) \\
\hline Production & \pm 3 \\
Fit & \pm 4 \\
Method & \pm 2 \\
\hline Total & \pm 5 \\
\hline
\end{tabular}

Contributions to the systematic uncertainty.

\section{Systematic uncertainty determination}

The systematic uncertainty was evaluated from a detailed study of different sources: the Monte Carlo production and detector simulation, the fitting procedure, and the possible intrinsic bias of the method.

The Monte Carlo correction function may be incorrect if the Monte Carlo poorly simulates the detector or the production characteristics of the events. For this reason we extract a systematic uncertainty due to the Monte Carlo simulation after a careful study of how well the simulation matches the data. We analyze effects from the momentum and transverse momentum of $\Xi_{c}^{0}$, the flavor (particle and antiparticle) of the $\Xi_{c}^{0}$, the decay mode of the $\Xi_{c}^{0}$, the multiplicity and position of the primary vertex, the error on the decay time and length, and the target region silicon strip detector (available for $2 / 3$ of the FOCUS data set). To obtain a systematic error, we split the data into statistically independent samples, on the basis of the production variables and of the mode. Then we compute the $\chi^{2} /$ d.o.f. for the hypothesis of consistency of the measurements. If we find $\chi^{2} /$ d.o.f. $>1$, we rescale the errors in order to have $\chi^{2} /$ d.o.f. $=1$, and extract the systematic error subtracting in quadrature the statistical error from the scaled error of the weighted average of the independent measurements. This method is based on the $S$-factor method of the Particle Data Group [1]. The only significant effects found are due to primary vertex multiplicity and $\Xi_{c}^{0}$ decay mode from which a systematic uncertainty of $\sigma$ (production $)= \pm 3$ fs is obtained.

The measurement we report is from a particular choice for the fit parameters and fitting technique (namely, the binned maximum likelihood technique). Varying these fitting conditions provides measurements which are all a priori likely. We calculate a systematic uncertainty by performing a set of lifetime measurements with different choices for the fitting parameters and the fitting technique. To study systematic effects related to our choice of fitting parameters we varied the location and width of the sidebands, the proper decay time fitting region and bin size. We also varied the correction function $f\left(t^{\prime}\right)$, by using a linear fit instead of the bin values. 
Since the proper time resolution (about 40 fs for the $\Xi^{-} \pi^{+}$mode and $80 \mathrm{fs}$ for the $\Omega^{-} K^{+}$mode) is close to the $\Xi_{c}^{0}$ lifetime, we decided to perform the measurement also with a convolved binned likelihood method [15] to check the fitting technique. In this method, the exponential decay is convolved with the smearing due to the time resolution. We used the Monte Carlo convolution correction function $F\left(t_{j}^{\text {gen }}, t_{i}^{\text {rec }}\right)$, which represents the probability of reconstructing an event in the decay time bin $i$ when the true decay time bin is $j$. The likelihood is constructed in the same way as explained before, but the number of expected events in the $i^{\text {th }}$ bin becomes:

$$
n_{i}=S \frac{\sum_{j} f\left(t_{j}^{\mathrm{gen}}, t_{i}^{\mathrm{rec}}\right) e^{-t_{j} / \tau}}{\sum_{i} \sum_{j} f\left(t_{j}^{\mathrm{gen}}, t_{i}^{\mathrm{rec}}\right) e^{-t_{j} / \tau}}+B \frac{b_{i}}{\sum_{i} b_{i}} .
$$

Instead of the reduced proper decay time, the proper decay time is fit. Fig. 4 shows the proper time distributions. The lifetime result of the convolved method is then considered as a further fit variant. The systematic uncertainty due to the fit variants is given by the variance of the set of measurements. We find $\sigma$ (fit) $= \pm 4$ fs.

The Monte Carlo simulation uses an input lifetime given by the current world average reported from the PDG [1] of $\tau\left(\Xi_{c}^{0}\right)=98$ fs. We investigate the range of good performance of the fitting method when the input lifetime for the correction function is $100 \mathrm{fs}$ with a mini Monte Carlo study. This consists of simulating a large number of samples with the same statistics as our data (both for signal and background events). We obtain events from exponential decaying populations with a given lifetime for the signal and for the background. We obtain the background lifetime by fitting the data sideband distributions to an exponential. For the signal events we study a wide range of lifetime values (from $10 \mathrm{fs}$ to $170 \mathrm{fs}$ ). Care is taken to account for the time resolution. The method works with an accuracy better than $10 \%$ for lifetimes greater than $55 \mathrm{fs}$. We study the accuracy in the range of the $\Xi_{c}^{0}$ measured lifetime, and measure a systematic uncertainty $\sigma$ (method) of \pm 2 fs. The mini Monte Carlo study also validates our estimate of the fit statistical error.

The total systematic uncertainty is obtained by adding in quadrature the contributions from the three independent sources. We thus find $\sigma(\mathrm{sys})= \pm 5 \mathrm{fs}$. See Table 1 for a summary of the contributions and the total systematic uncertainty.

\section{Conclusions}

We have measured the lifetime of the charmed baryon $\Xi_{c}^{0}$, fully reconstructing the decays $\Xi_{c}^{0} \rightarrow \Xi^{-} \pi^{+}$, and $\Xi_{c}^{0} \rightarrow \Omega^{-} K^{+}$. Figure 5 a shows the invariant 
mass plot for the combined sample. From the $110 \pm 17$ reconstructed events we measure $\tau\left(\Xi_{c}^{0}\right)=118_{-12}^{+14}$ (stat) \pm 5 (sys) fs. Figure $5 \mathrm{~b}$ shows the Monte Carlo corrected sideband subtracted proper time distribution for the combined sample for the signal region and sideband region. This measurement greatly improves upon the accuracy of previous measurements, reducing the percentage error on the $\Xi_{c}^{0}$ lifetime from $20 \%$ to $10 \%$.

\section{Acknowledgements}

We wish to acknowledge the assistance of the staffs of Fermi National Accelerator Laboratory, the INFN of Italy, and the physics departments of the collaborating institutions. This research was supported in part by the U. S. National Science Foundation, the U. S. Department of Energy, the Italian Istituto Nazionale di Fisica Nucleare and Ministero dell'Università e della Ricerca Scientifica e Tecnologica, the Brazilian Conselho Nacional de Desenvolvimento Científico e Tecnológico, CONACyT-México, the Korean Ministry of Education, and the Korean Science and Engineering Foundation.

\section{References}

[1] Particle Data Group, D. E. Groom et al., Eur. Phys. J. C 15 (2000) 1.

[2] G. Bellini, I. I. Y. Bigi, and P. J. Dornan, Phys. Rept. 289 (1997) 1.

[3] B. Guberina and B. Melic, Eur. Phys. J. C2 (1998) 697.

[4] FOCUS Collaboration, J. M. Link et al., Phys. Rev. Lett. 88 (2002) 161801.

[5] CLEO Collaboration, A. H. Mahmood et al., Phys. Rev. Lett. 86 (2001) 2232.

[6] SELEX Collaboration, A. Kushnirenko et al., Phys. Rev. Lett. 86 (2001) 5243.

[7] FOCUS Collaboration, J. M. Link et al., Phys. Lett. B 523 (2001) 53.

[8] ACCMOR Collaboration, S. Barlag et al., Phys. Lett. B 236 (1990) 495.

[9] E687 Collaboration, P. L. Frabetti et al., Nucl. Instrum. Meth. A 320 (1992) 519.

[10] E687 Collaboration, P. L. Frabetti et al., Phys. Rev. Lett. 70 (1993) 2058.

[11] G. Bellini et al., Nucl. Instrum. Meth. A 252 (1986) 366.

[12] FOCUS Collaboration, J. M. Link et al., submitted to Nucl. Instrum. Meth., hep-ex/0204023. 
[13] FOCUS Collaboration, J. M. Link et al., Nucl. Instrum. Meth. A 484 (2002) 270.

[14] FOCUS Collaboration, J. M. Link et al., Nucl. Instrum. Meth. A 484 (2002) 174.

[15] E687 Collaboration, P. L. Frabetti et al., Phys. Lett. B 357 (1995) 678. 

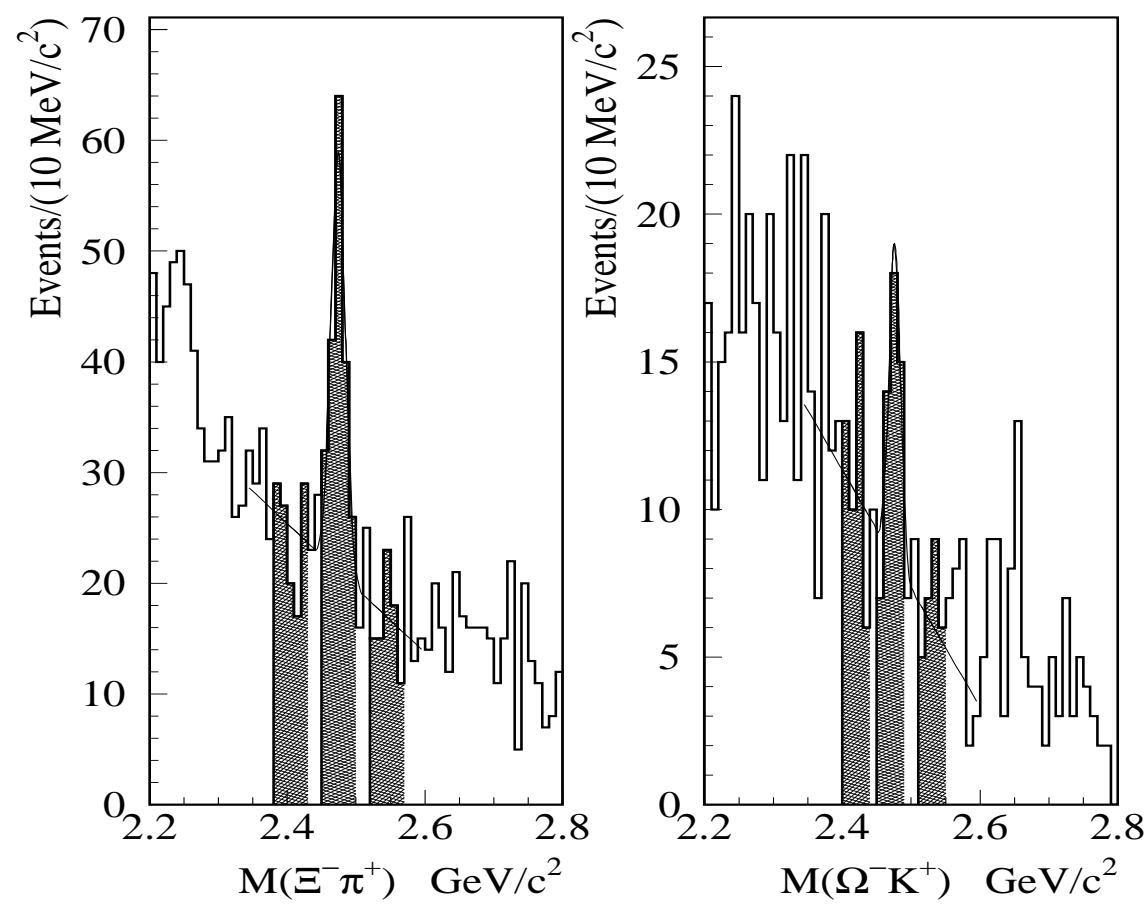

Fig. 1. Invariant mass distributions for each mode. The fit uses a Gaussian for the signal and a straight line for the background. The signal region is defined by the central shaded region. The sideband region, defined by the two symmetrical shaded regions, contains the events used to reproduce the decay time of the background events in the signal region. 

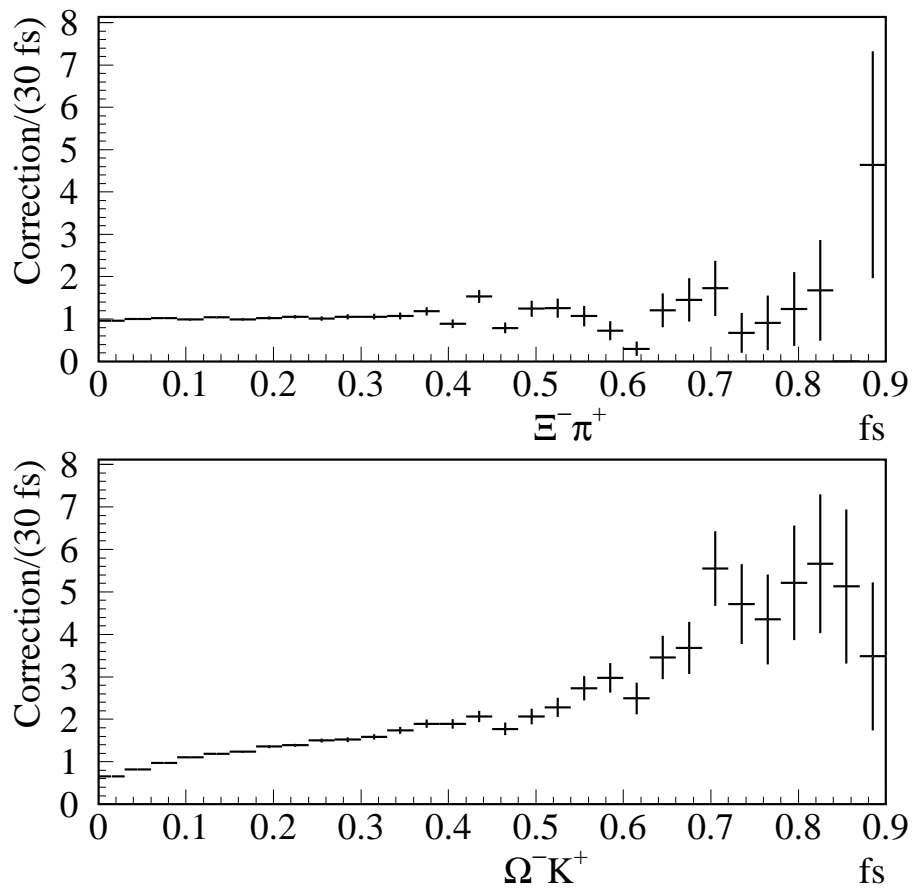

Fig. 2. Monte Carlo correction function $f\left(t^{\prime}\right)$ for each decay mode.
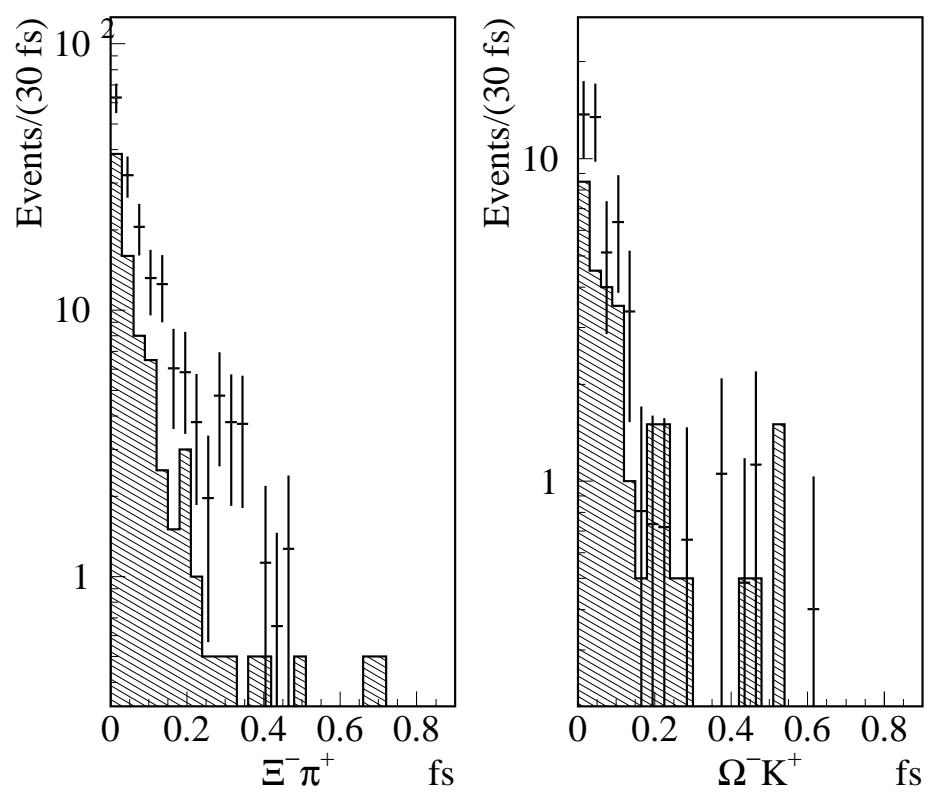

Fig. 3. Reduced proper time distributions, corrected by the Monte Carlo correction function, for each decay mode in the signal region and the sideband region (shaded). 

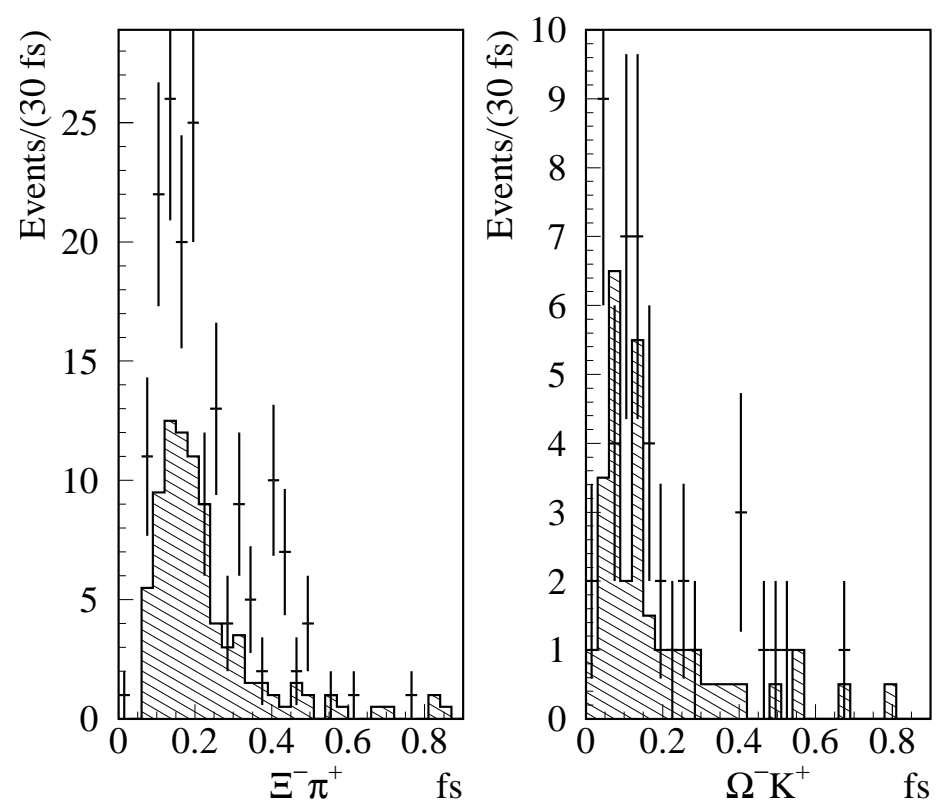

Fig. 4. Proper time distribution for the two decay modes in the signal region and sideband region (shaded). 

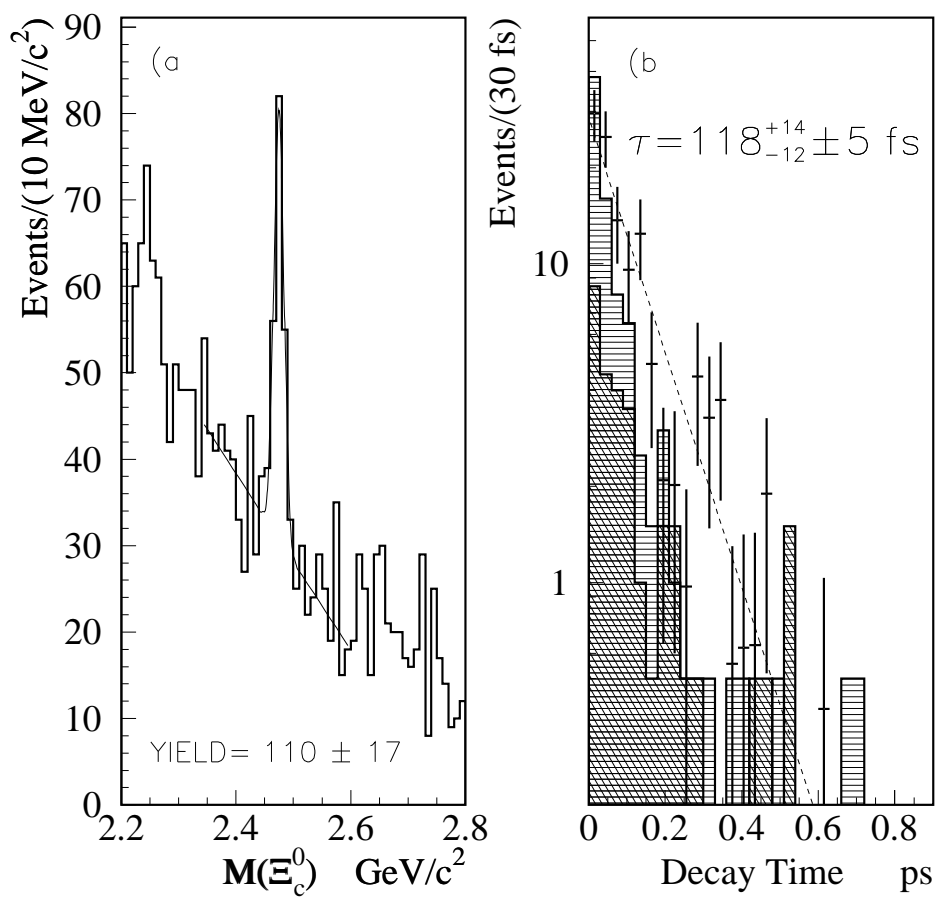

Fig. 5. Mass and lifetime distributions for the combined sample. a) Invariant mass distribution; fit with a Gaussian for the signal and a first order polynomial for the background. b) Monte Carlo corrected and sideband subtracted reduced proper time distribution for the signal region (points). The histograms with different shades are the time distributions for the sidebands for the two decay modes (lighter shade: $\Xi^{-} \pi^{+}$, darker shade: $\left.\Omega^{-} K^{+}\right)$. The dashed line shows the result of the fit. 